\title{
A correlated electron diffraction, in situ neutron diffraction and dielectric properties investigation of poled (1-x) $\mathrm{Bi}_{0.5} \mathrm{Na}_{0.5} \mathrm{TiO}_{3}-x \mathrm{BaTiO}_{3}$ ceramics
}

\author{
Jian Wang, ${ }^{1}$ Yun Liu, ${ }^{1, a)}$ Ray L. Withers, ${ }^{1}$ Andrew Studer, ${ }^{2}$ Qian Li, ${ }^{1}$ Lasse Norén, ${ }^{1}$ \\ and Yiping Guo $\mathrm{O}^{1,3}$ \\ ${ }^{1}$ Research School of Chemistry, The Australian National University, Canberra, ACT0200, Australia \\ ${ }^{2}$ Bragg Institute, Australian Nuclear Science and Technology Organisation (ANSTO), Lucas Heights, \\ NSW 2232, Australia \\ ${ }^{3}$ State Key Laboratory of MMCs, Shanghai Jiaotong University, Shanghai 200240, People's Republic of China
}

(Received 8 August 2011; accepted 24 September 2011; published online 25 October 2011)

\begin{abstract}
A correlated electron diffraction, temperature-dependent in situ neutron diffraction, and temperature-dependent dielectric properties investigation of poled $(1-x) \mathrm{Bi}_{0.5} \mathrm{Na}_{0.5} \mathrm{TiO}_{3}-x \mathrm{BaTiO}_{3}$ $($ BNTBT100x $)(x=0.04,0.07$, and 0.12$)$ samples has been carried out. The results show that the depolarization temperature, $T_{\mathrm{d}}$, of the rhombohedral BNTBT 4 sample is associated with the disappearance of $\mathbf{G} \pm 1 / 2[111]^{*}$ p satellite reflections and $a^{-} a^{-} a^{-}$octahedral tilting while that of the BNTBT 12 sample is associated with a metrically tetragonal to metrically cubic or pseudo-cubic symmetry. In the case of the poled BNTBT 7 sample in the MPB region, the dielectric properties show a quite distinct two stage transition from a room temperature clearly metrically tetragonal phase again to a metrically cubic or pseudo-cubic symmetry above $150{ }^{\circ} \mathrm{C}$. There is no apparent change in its average structure in vicinity of $\mathrm{T}_{\mathrm{d}}$ in BNTBT 7. Electron diffraction shows the presence of considerable octahedral tilt twin disorder in all three samples. (C) 2011 American Institute of Physics. [doi:10.1063/1.3654140]
\end{abstract}

\section{INTRODUCTION}

As a promising lead-free piezoelectric material, the synthesis and crystal growth conditions, ${ }^{1-4}$ effect of doping, ${ }^{4,5}$ local micro-structure ${ }^{1,6-9}$ and piezoelectric properties ${ }^{1,10,11}$ of the $(1-x) \mathrm{Bi}_{0.5} \mathrm{Na}_{0.5} \mathrm{TiO}_{3}-x \mathrm{BaTiO}_{3}$ (abbreviated as $\mathrm{BNTBT100x}$ hereafter) solid solution system have been extensively studied. It is well known that properties such as the piezoelectric constant $d_{33}$, the electro-mechanical coupling coefficient $k_{\mathrm{p}}$, and the electric field induced strain response are optimal in the vicinity of a so-called morphotropic phase boundary (MPB) transition region from a largely rhombohedral $R 3 c$ to a tetragonal $P 4 \mathrm{~mm}$ (or $P 4 \mathrm{bm}$ ) symmetry in this system, at around $\mathrm{x} \sim 0.06-0.08$. For instance, a $k_{\mathrm{p}} \sim 36.7 \%,{ }^{11}$ a $d_{33}$ of $176 \mathrm{pC} / \mathrm{N},{ }^{12}$ and a high strain response of $0.42 \%$ (Ref. 1) can only be achieved in this MPB region. Much research ${ }^{1,4,13-15}$ has therefore focused on the relation between structure (both average as well as local) and the associated piezoresponse properties of BNTBTs in this MPB region.

In a recent study of the BNTBT system from $x=0.04$ to $\mathrm{x}=0.10$, the antiferroelectric-like behavior and a large, electric field induced, strain response was observed at room temperature in BNTBT 7, corresponding to the composition with the lowest depolarization temperature $\left(T_{\mathrm{d}}\right) .{ }^{1}$ Note that the piezoelectric response of polycrystalline ceramics can only be fully activated after a poling process that also introduces an observable depolarization temperature $T_{\mathrm{d}}$ in the dielectrictemperature spectra. Experimentally the closer to the MPB region, the lower the $T_{\mathrm{d}}$ and the higher the measured strain response. ${ }^{1}$ Exploring the nature of this depolarization temper-

\footnotetext{
a) Author to whom correspondence should be addressed. Electronic mail: yliu@rsc.anu.edu.au.
}

ature, $T_{\mathrm{d}}$, and its relationship with the average as well as the local structure is therefore critical to further optimization of the piezoelectric properties of this material for practical applications and is a major focus of this paper.

Previous structural work on the BNTBT system has shown, in addition to the well-defined set of parent perovskite Bragg reflections $\mathbf{G}$, the presence of considerable, inherent structural disorder as indicated by the co-existence of $\mathbf{G} \pm 1 / 2[111]^{*}$ (subscript $\mathrm{p}$ for parent perovskite sub-structure) and typically broad $\mathbf{G} \pm 1 / 2[110]^{*}$ " "satellite reflections" $" 1,15,16$ as well as essentially continuous diffuse streaking linking the two over a wide composition range, both across and on either side of the MPB region. The relative intensities of these "satellite reflections" change dramatically with composition. On the rhombohedral side of the MPB region, the $\mathbf{G} \pm 1 / 2[111]{ }_{\mathrm{p}}$ satellite reflections dominate while on the nominally tetragonal side, the still broad $\mathbf{G} \pm 1 / 2\langle 110\rangle{ }^{*}$ satellite reflections dominate.

Daniels et $a l .{ }^{13}$ has previously reported an electric field induced, metrically pseudo-cubic to tetragonal phase transition in both BNTBT 6 and 7. In this work, we aim to investigate the correlation between the depolarization temperature $\left(T_{\mathrm{d}}\right)$, polarization relaxation, and associated electric field-induced microstructural evolution by a combination of electron diffraction, in situ neutron diffraction and the temperature-dependent dielectric properties of BNTBT 4, 7, and 12 samples.

\section{EXPERIMENTAL}

BNTBT 4, 7, and 12 ceramics were synthesized by solid state reaction. High purity $\mathrm{Na}_{2} \mathrm{CO}_{3}, \mathrm{Bi}_{2} \mathrm{O}_{3}, \mathrm{BaCO}_{3}$, and nano- $\mathrm{TiO}_{2}(\sim 20 \mathrm{~nm})$ were used as starting materials. The starting materials were weighed and mixed thoroughly in a 
planetary ball mill for $5 \mathrm{~h}$ using ethanol as a medium. The mixed powders were then dried, ground, and calcined at $800^{\circ} \mathrm{C}$ for $3 \mathrm{~h}$. The calcined powder was then ball-milled again for $2 \mathrm{~h}$ and dried, followed by the addition of poly(vinylalcohol) as a binder. The resultant powder was then granulated and pressed into pellets with a diameter of $13 \mathrm{~mm}$ under a uniaxial pressure of $200 \mathrm{MPa}$. These pellets were then sintered in air at $1150{ }^{\circ} \mathrm{C}$ for $2 \mathrm{~h}$ in a covered alumina crucible. Finally, the as-sintered ceramic pellets were polished down to $0.5 \mathrm{~mm}$ thickness and coated with silver paste on their surfaces and heat treated at $500^{\circ} \mathrm{C}$ for $5 \mathrm{~min}$ to ensure good electrical contact. Grids suitable for transmission electron microscopy were prepared by crushing the samples in acetone and dispersing them onto lacey carbon coated grids. Electron diffraction patterns (EDPs) were obtained using a Philips EM 430 TEM operating at $300 \mathrm{kV}$.

Neutron powder diffraction data from these pelleted samples were collected in situ as a function of applied electric field and temperature at a wavelength of $1.7276 \AA$ using Wombat, the high intensity neutron diffraction beamline at ANSTO. A schematic of the experimental set-up used is shown in Fig. 1. Each sample was fixed to a central stage and conducting wires attached to the silver coatings on either side of the sample to enable a voltage to be applied. Data was first collected at room temperature under applied voltages of $0 \mathrm{kV}$, $1.5 \mathrm{kV}$, and then back to $0 \mathrm{kV}$, respectively. Data was then collected at selected temperatures under the same bias of $0.5 \mathrm{kV}$ (to enhance the depolarization effect during characterization). For each temperature and voltage point, the same procedure was followed: the sample was initially put in the parallel position $\left(\omega=0^{\circ}\right)$ to the incident neutron beam. The direction of the applied electric field is then perpendicular to both the beam and the sample. The central stage was then rotated anti-clock wise around the normal direction and patterns were collected in rotation angle $\omega$ increments of $30^{\circ}$. The data at all $\omega$ rotation angles were then added together to minimize the effect of field induced changes in texture and to produce the diffraction patterns presented hereafter.

\section{RESULTS AND DISCUSSION}

\section{A. Electron diffraction}

The BNTBT samples were first investigated by electron diffraction at room temperature and show, in addition to a

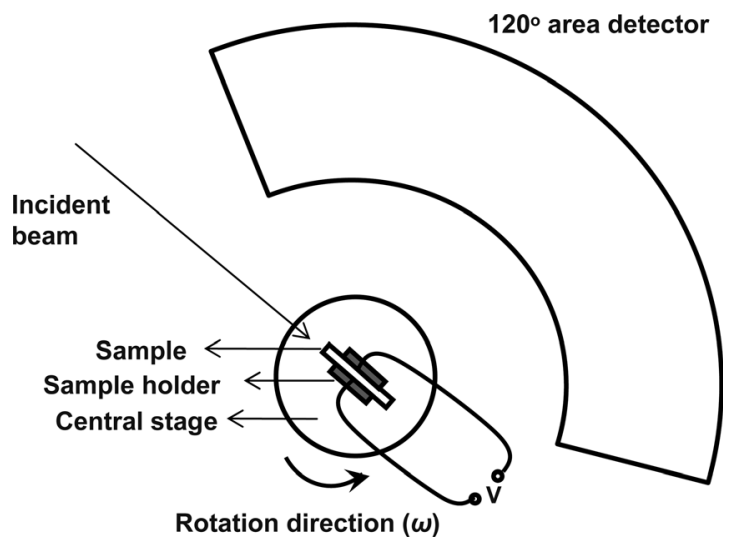

FIG. 1. The schematic of the in situ neutron diffraction experiment.

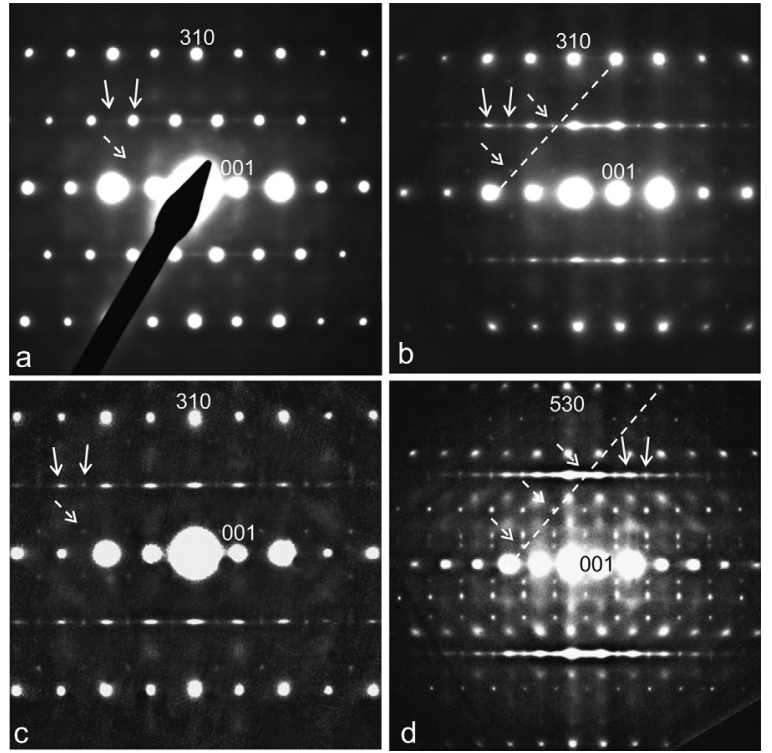

FIG. 2. The $\langle-130\rangle_{\mathrm{p}}$ zone axis electron diffraction patterns (EDPs) of BNTBT 4 (a), BNTBT 7 (b), and BNTBT 12 (c) as well as of BNTBT 7 along the $\langle-350\rangle_{\mathrm{p}}$ zone axis $(\mathrm{d})$.

well-defined set of parent perovskite Bragg reflections $\mathbf{G}$, the presence of considerable, inherent structural disorder as indicated by the co-existence of $\mathbf{G} \pm 1 / 2\left[111{ }^{*}\right.$ p (subscript $p$ for parent perovskite sub-structure) and typically streaked out $\mathbf{G} \pm 1 / 2[110]{ }^{*}$ "satellite reflections" ${ }^{" 1,15,16}$ as well as close to continuous diffuse streaking linking the two over a wide composition range, both across and on either side of the MPB region. Figures 2(a)-2(c), for example, show room temperature $\langle-130\rangle_{\mathrm{p}}$ zone axis electron diffraction patterns (EDPs) typical of the as-synthesized BNTBT 4, 7, and 12 samples while Fig. 2(d) shows a typical $\langle-350\rangle_{\mathrm{p}}$ zone axis EDP of the BNTBT 7 sample.

Note the co-existence of $\mathbf{G} \pm 1 / 2[111]_{\mathrm{p}}{ }^{*}$ and $\mathbf{G} \pm 1 / 2[110]_{\mathrm{p}} *$ type 'satellite reflections' in each EDP (solid arrows in Fig. 2) as well as essentially continuous diffuse streaking linking the two. Note also that the relative intensities of these "satellite reflection" types change dramatically with composition. On the rhombohedral side of the MPB region, the $\mathbf{G} \pm 1 / 2[111]^{*}$ satellite reflections dominate (see Fig. 2(a)) while on the nominally tetragonal side, the $\mathbf{G} \pm 1 / 2\langle 110\rangle^{*}$ p satellite reflections dominate (see Fig. 2(c)). The dominant $\mathbf{G} \pm 1 / 2[111]_{\mathrm{p}} *$ type satellite reflections of the rhombohedral $R 3 c$ side of the MPB region (see Fig. 2(a)) are known to originate from an $a^{-} a^{-} a^{-}$octahedral tilt distribution while the $\mathbf{G} \pm 1 / 2[110]_{\mathrm{p}} *$ type satellite reflections of the tetragonal side of the MPB region are presumed to originate from an $a^{0} a^{0} c^{+}$octahedral tilt distribution.

Note also the presence of generally weak but always present $\mathbf{G} \pm^{(2 \mathrm{~m}+1)} / 6[313]_{\mathrm{p}} *$ type 'satellite reflections' (dashed arrows in Fig. 2(b)) corresponding to $\mathbf{G} \pm 1 / 6[313]_{\mathrm{p}} *$ (or $\left.\mathbf{G} \pm\left[1 / 2,1 / 6,{ }^{1} / 2\right]_{\mathrm{p}} *\right)$ and $\mathbf{G} \pm{ }^{3} / 6[313]_{\mathrm{p}} *$ or $\mathbf{G} \pm 1 / 2[111]_{\mathrm{p}} *$ type satellite reflections in Fig. 2(b) and the $\mathbf{G} \pm^{(2 \mathrm{~m}+1)} / 10[535]_{\mathrm{p}}$ * or $\left.\mathbf{G} \pm(2 \mathrm{~m}+1)\left[1 / 2,{ }^{3} / 10,1 / 2\right]_{\mathrm{p}} *\right)$ type "satellite reflections" (dashed arrows in Fig. 2(d)). These apparent "reflections" are in fact simply cuts through rods of diffuse intensity of $\mathbf{G} \pm[1 / 2, \xi, 1 / 2]_{\mathrm{p}} *$ ( $\xi$ continuous) type and can only be 

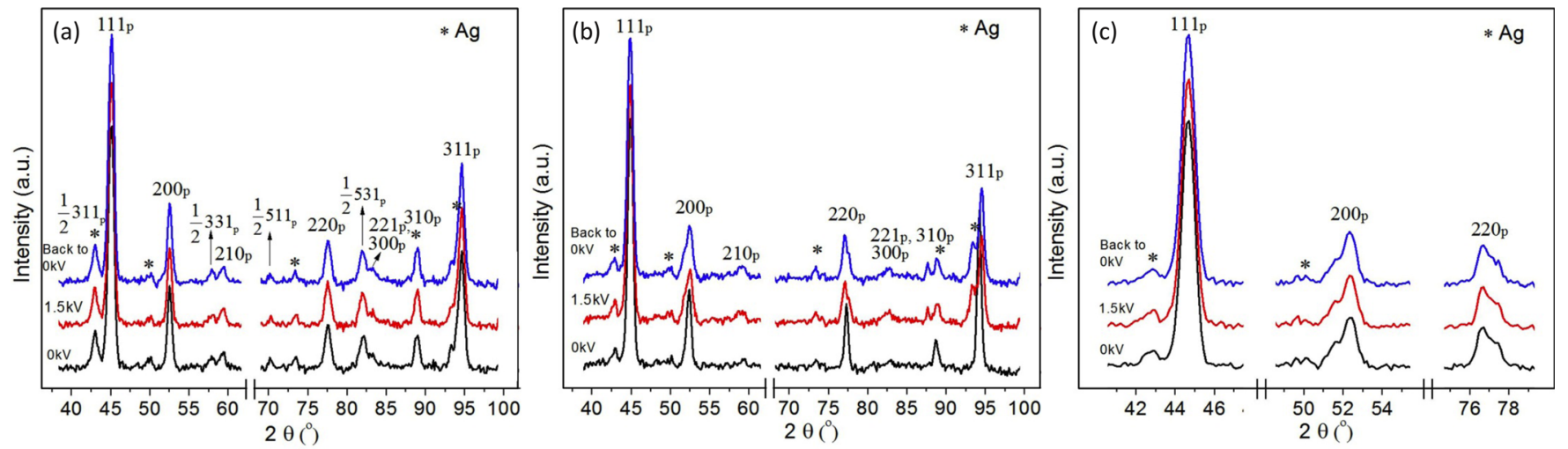

FIG. 3. (Color online) The in situ neutron diffraction patterns of BNTBT 4 (a), BNTBT 7 (b), and BNTBT 12 (c) collected at room temperature under applied electric fields of $0 \mathrm{KV}$ (the initial state), $1.5 \mathrm{KV}$, and $0 \mathrm{KV}$ (after the withdrawal of the electric field).

associated with additional octahedral tilting around the $[010]_{\mathrm{p}}$ direction just as the $\mathbf{G} \pm[1 / 2,1 / 2, \xi]_{\mathrm{p}}$ * rods immediately apparent in Fig. 2 can only be associated with additional octahedral tilting around the $[001]_{\mathrm{p}}$ direction. Clearly there is considerable scope for octahedral tilt twin disorder in the BNTBT system. It is important to note that such octahedral tilt twin disorder locally creates a distinctly different environment for the $\mathrm{Na} / \mathrm{Bi} / \mathrm{Ba}$ ions at the twin interface and hence the probability of a distinctly different local direction of polarization.

\section{B. Room temperature neutron diffraction under an applied electric field}

Figure 3 shows selected portions of the room temperature neutron diffraction patterns (NDPs) of the BNTBT 4, 7, and 12 ceramic samples at $0 \mathrm{kV}, 1.5 \mathrm{kV}$, and upon returning to $0 \mathrm{kV}$. The patterns are indexed with respect to the underlying parent perovskite sub-structure (labeled with the subscript $p$ ) in each case while regions where the silver electrode may make a small contribution to the observed intensity are marked with an asterisk in each case.

The unambiguous presence of $\mathbf{G} \pm 1 / 2[111]_{\mathrm{p}} *$ type reflections such as $1 / 2\langle 331\rangle_{\mathrm{p}}{ }^{*}, 1 / 2\langle 511\rangle_{\mathrm{p}}{ }^{*}$, and $1 / 2\langle 531\rangle_{\mathrm{p}}{ }^{*}$ in the BNTBT 4 sample (see Fig. 3(a)) is consistent with the presence of $a^{-} a^{-} a^{-}$octahedral tilting and suggests that the BNTBT 4 sample is in the rhombohedral $R 3 c$ ferroelectric phase at room temperature, as expected. Note that there is no clear rhombohedral splitting of the parent perovskite reflections within the resolution available to us and that the applied electric field of $1.5 \mathrm{kV}$ has no obvious effect upon the neutron diffraction pattern. For the BNTBT 12 sample (see Fig. 3(c)), the clear $\sim 1: 2$ splittings of the parent $\langle 200\rangle_{\mathrm{p}}{ }^{*}$ and $\langle 220\rangle_{\mathrm{p}}{ }^{*}$ peaks but not of the parent $\langle 111\rangle_{\mathrm{p}}{ }^{*}$ peak confirms that BNTBT 12 is already in a metrically tetragonal phase at room temperature before the electric field is applied, again as expected. No evidence could be found for a $\mathbf{G} \pm 1 / 2$ $\langle 110\rangle_{\mathrm{p}} *$ type satellite reflection, which is perhaps not surprising given the broadness of such peaks apparent from electron diffraction (see Fig. 2(c)). As for the BNTBT 4 sample, the applied electric field again has no obvious effect upon the neutron powder pattern.

In the case of the BNTBT 7 sample, however, the initial $0 \mathrm{kV}$ diffraction pattern is either metrically cubic or at least pseudo-cubic as there is no profile shape asymmetry or obvious splitting of any of the observed parent reflections. The lattice parameters for this metrically cubic phase are $\mathrm{a}=3.9059$ (3) $\AA$ and $\mathrm{V}=58.586$ (12) $\AA^{3}$. By $1.5 \mathrm{kV}$, however, there has been a clear electric field induced transition to a metrically tetragonal phase with the lattice parameters of $a=3.890$ (3) $\AA, c=3.938(4) \AA$, and $\mathrm{V}=59.59(9) \AA^{3}$, as is apparent from the characteristic splitting of the parent $\langle 200\rangle_{\mathrm{p}}{ }^{*}$ and $\langle 220\rangle_{\mathrm{p}}{ }^{*}$ peaks but not of the parent $\langle 111\rangle_{\mathrm{p}}{ }^{*}$ peak (see Fig. 3(b)). The lattice parameter $c$ value in metrically tetragonal phase of the poled BNTBT 7 is significantly higher than the $a$ value in metrically cubic phase of the corresponding unpoled sample, suggesting the high strain is induced by an external electrical field. This metrically tetragonal symmetry then remains upon lowering of the applied electric field back to zero. The electric field induced peak splitting is quite similar in fact to that observed for the BNTBT 12 sample and implies parent lattice parameters $a_{\mathrm{p}}=b_{\mathrm{p}}<c_{\mathrm{p}}$. This result is consistent with previous reports for the behavior of BNTBT 7 (Refs. 7 and 8) under an applied electric field. It differs significantly though from the behavior recently reported for a $\left(\mathrm{Bi}_{0.5} \mathrm{Na}_{0.5} \mathrm{TiO}_{3}\right)_{0.92}(\mathrm{Ba}-$ $\left.\mathrm{TiO}_{3}\right)_{0.06}\left(\mathrm{~K}_{0.5} \mathrm{Na}_{0.5} \mathrm{NbO}_{3}\right)_{0.02}$ sample, which underwent instead a reversible phase transition from a pseudo-cubic phase to the rhombohedral $R 3 c$ ferroelectric phase under an applied electric field. ${ }^{17}$

\section{Dielectric spectra and in situ high temperature neutron diffraction}

Figure 4(a) shows the dielectric constant and loss tangent of BNTBT 4 as a function of temperature at three separate frequencies while Fig. 4(b) shows selected portions of the corresponding in situ neutron powder diffraction patterns of a $0.5 \mathrm{kV}$ biased, pre-poled, BNTBT 4 sample collected at different temperatures up to $200^{\circ} \mathrm{C}$. The depolarization temperature $\left(T_{\mathrm{d}}\right)$, corresponding to the first inflection point or peak in the dielectric loss tangent curve, occurs at a temperature of $\sim 162^{\circ} \mathrm{C}$ in BNTBT 4 (see Fig. 4(a)). Below $T_{\mathrm{d}}$, almost no frequency dependence of the dielectric constant curves is apparent. Above the sharp jump of the dielectric constant at $T_{\mathrm{d}}$, the dielectric constant spectra shows noticeable dispersion, i.e., frequency dependence, demonstrating significant polarization relaxation above $T_{\mathrm{d}}$. 

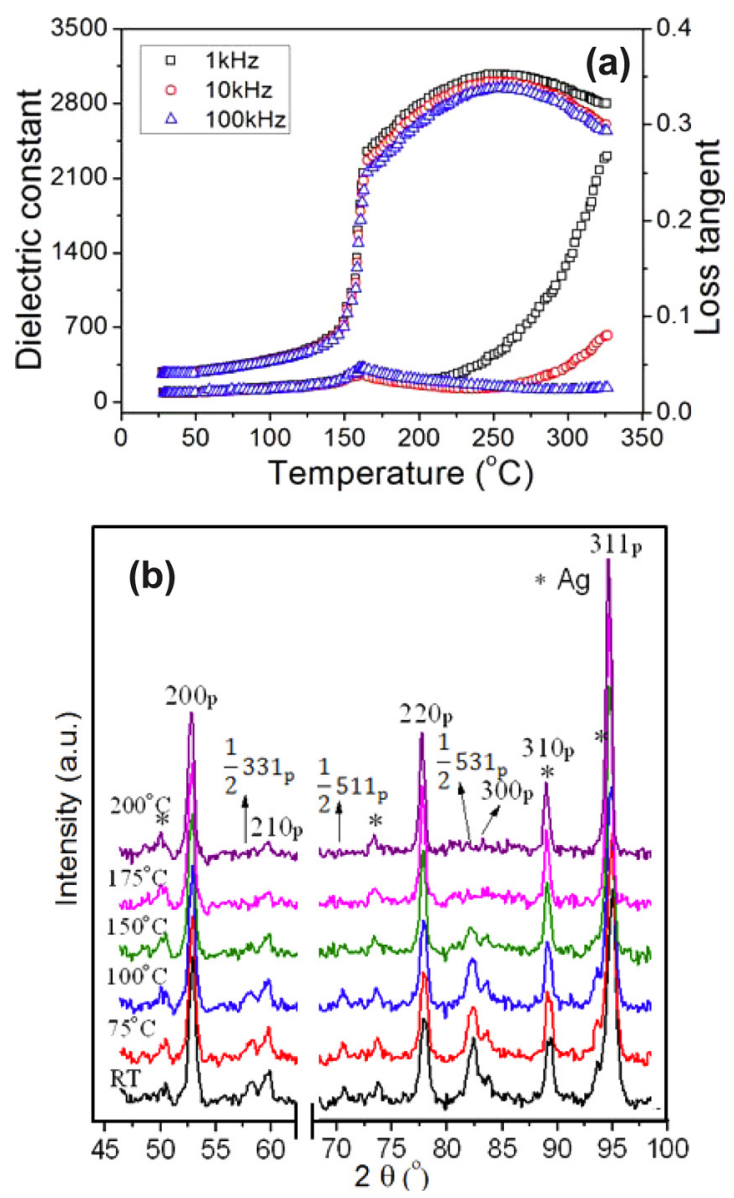

FIG. 4. (Color online) Dielectric spectra (a) and neutron diffraction patterns (b) of BNTBT 4 ceramics collected at different temperatures.

This transition in dielectric properties correlates very well with the disappearance of the $\mathbf{G} \pm 1 / 2[111]_{\mathrm{p}} *$ type reflections characteristic of the rhombohedral ferroelectric phase such as $1 / 2\langle 331\rangle_{\mathrm{p}} *, 1 / 2\langle 511\rangle_{\mathrm{p}} *$, and $1 / 2\langle 531\rangle_{\mathrm{p}}{ }^{*}$ between the $150{ }^{\circ} \mathrm{C}$ and $175^{\circ} \mathrm{C}$ curves in Fig. 4(b). The transition thus corresponds to the rhombohedral ferroelectric to so-called anti-ferroelectric (AFE) structural phase transition reported in the literature. Note that the intensity of the $1 / 2\langle 331\rangle_{\mathrm{p}}{ }^{*}, 1 / 2\langle 511\rangle_{\mathrm{p}}{ }^{*}$, and $1 /{ }_{2}\langle 531\rangle_{\mathrm{p}}{ }^{*}$ satellite reflections characteristic of the rhombohedral phase decrease systematically with increasing temperature, particularly noticeable between the $100{ }^{\circ} \mathrm{C}$ and $150^{\circ} \mathrm{C}$ curves. The depolarization temperature $\left(T_{\mathrm{d}}\right)$ in the BNTBT 4 sample therefore reflects a structural phase transition from a rhombohedral ferroelectric to a metrically pseudo-cubic, so-called AFE phase, which shows relaxor characteristics in the clear frequency dependence of the dielectric constant. The long range ordered rhombohedral ferroelectric phase, by contrast, does not show dispersive behavior in its dielectric spectra (see Fig. 4(a)).

In the case of the BNTBT 12 sample, $T_{\mathrm{d}}$ is observed at $\sim 180^{\circ} \mathrm{C}$ (see Fig. 5(a)). Selected portions of the in situ neutron powder diffraction patterns of a $0.5 \mathrm{kV}$ biased BNTBT 12 sample collected at different temperatures up to $200^{\circ} \mathrm{C}$ are shown in Fig. 5(b). The transition in dielectric properties again correlates well with a subtle change in metric symmetry, from metrically tetragonal below $180{ }^{\circ} \mathrm{C}$ to metrically cubic (or pseudo-cubic) above, as is apparent from the disap-
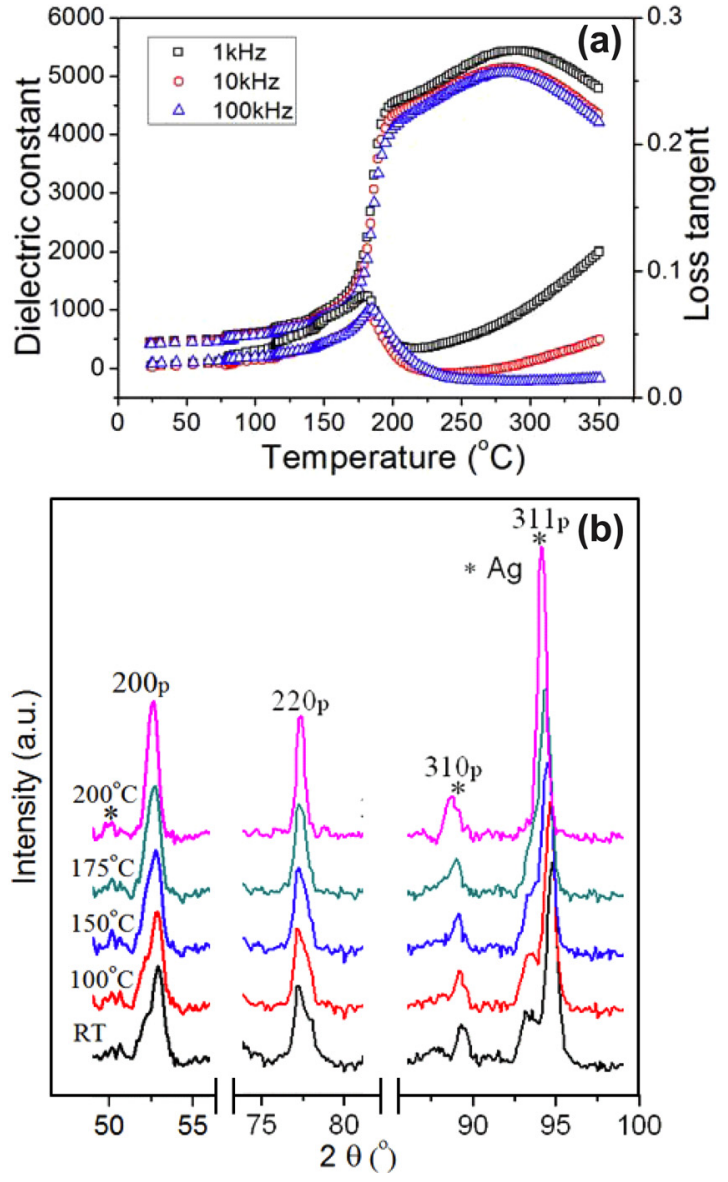

FIG. 5. (Color online) Dielectric spectra (a) and neutron diffraction patterns (b) of BNTBT 12 ceramics collected at different temperatures.

pearance of the shoulders to the $\langle 002\rangle_{\mathrm{p}}{ }^{*}$ and $\langle 220\rangle_{\mathrm{p}}{ }^{*}$ peaks in the $200{ }^{\circ} \mathrm{C}$ plot. $T_{\mathrm{d}}$ in the case of BNTBT 12 sample is thus related to a structural phase transition from a metrically tetragonal, nominally ferroelectric phase to the metrically pseudo-cubic, so-called AFE relaxor phase. ${ }^{5}$ Note that relaxor behavior is observed both below as well as above $T_{\mathrm{d}}$ in this case.

Finally, Fig. 6(a) shows the temperature and frequency dependence of the dielectric constant and loss tangent of the BNTBT 7 sample. It is intriguing that this MPB sample behaves in a different fashion to the other two, non-MPB samples, in particular there is a much smaller jump in the dielectric constant at a much lower $T_{\mathrm{d}}$ of $\sim 70{ }^{\circ} \mathrm{C}$. Above $T_{\mathrm{d}}$ there is then a steady increase in dielectric constant until a rather broad shoulder in the dielectric constant curves and a corresponding change in slope of the dielectric loss curves at $\sim 150{ }^{\circ} \mathrm{C}$ well below $T_{\mathrm{m}} \sim 270^{\circ} \mathrm{C}$ (the temperature at which the dielectric constant peaks). Below $T_{\mathrm{d}}$, the dielectric constant curves show little dispersion. Above $T_{\mathrm{d}}$ and up to $\sim 200^{\circ} \mathrm{C}$, however, the dielectric dispersion increases significantly before narrowing again up to $T_{\mathrm{m}}$.

From the structural point of view, the poled BNTBT 7 sample has an electric field triggered, metrically tetragonal phase at room temperature (Fig. 3 and Fig. 6(a)). There is no immediately obvious difference between the room temperature pattern and that at $75^{\circ} \mathrm{C}$ (above $T_{\mathrm{d}}$ ) or $100^{\circ} \mathrm{C}$ until the $150{ }^{\circ} \mathrm{C}$ pattern at which point there is a clear transition to a 

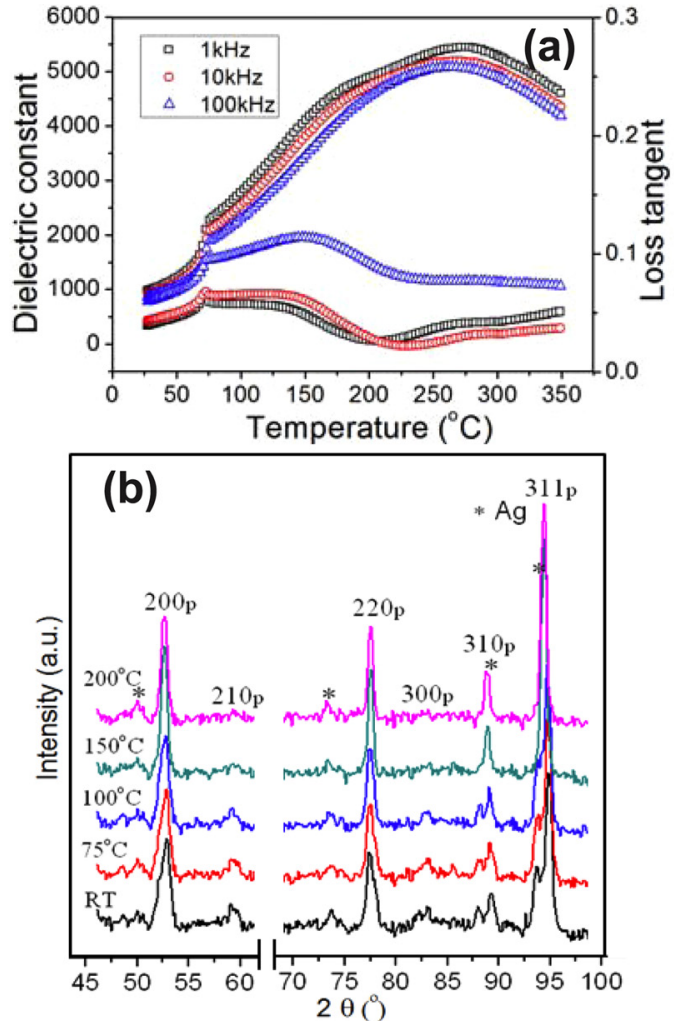

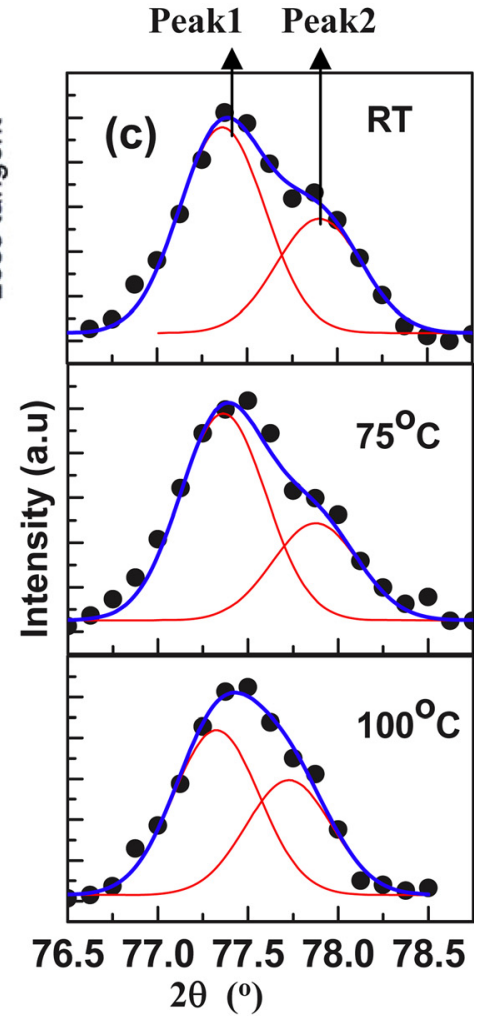

FIG. 6. (Color online) Dielectric spectra (a) and neutron diffraction patterns (b) of BNTBT 4 ceramics collected at different temperatures. (c) presents the Gaussian fitting result of a typical $(220)_{\mathrm{p}}$ peaks at different temperatures. metrically cubic or pseudo-cubic phase (as shown by the sudden merging of the previously split $\langle 013\rangle_{\mathrm{p}}{ }^{*}$ and $\langle 113\rangle_{\mathrm{p}}{ }^{*}$ peaks and the disappearance of shoulders on the $\langle 002\rangle_{\mathrm{p}}{ }^{*}$ and $\langle 220\rangle_{\mathrm{p}}{ }^{*}$ peaks at $150^{\circ} \mathrm{C}$ ). The in situ neutron diffraction data thus suggests a phase transition from a metrically tetragonal to a metrically cubic or pseudo-cubic phase somewhere in the 100 to $150{ }^{\circ} \mathrm{C}$ temperature range. There is, however, no obvious change in the average structure in the vicinity of $T_{\mathrm{d}}$. Figure 6(c) shows the Gaussian fitting results of a typical $\langle 220\rangle_{\mathrm{p}}{ }^{*}$ peaks collected at room temperature, $75^{\circ} \mathrm{C}$ and $100{ }^{\circ} \mathrm{C}$, respectively. It is no doubt that this parent peak always consists of two peaks (refer to Fig. 6(c)) due to the existence of the electrical field induced metrically tetragonal phase. The peak 1 always stays at the same with a $2 \theta$ of $\sim 77.36^{\circ}$ but the peak 2 shifts as a function of temperature ( $\sim 77.90^{\circ}$ at room temperature, $77.76^{\circ}$ at $75^{\circ} \mathrm{C}$ and $\sim 77.72^{\circ}$ at $100^{\circ} \mathrm{C}$ ), suggesting more significant structural change occurred along the parent $c$ direction. This is easily understood due to the octahedral tilt twin disorder where two types of octahedral tilting co-occur either around the $\langle 111\rangle \mathrm{p}$ direction or around the[001]p direction, respectively. This sort of local structure contributes to the profile distortion observed in their diffraction peak but does not considerably changed the average structure. In this case, the $T_{d}$ is probably the balancing sequence between the local structures and parent structure matrix, which cannot be observed via the neutron diffraction technique - as it only reflects the average structure. Another possibility is that the observed $T_{\mathrm{d}}$ in BNTBT 7 possibly reflects the onset of a minority amount of the metrically cubic phase but the transition does not appear to be completed until $\sim 150{ }^{\circ} \mathrm{C}$.

It is noteworthy that for each of the BNTBT 4, 7, and 12 samples, the metric symmetry becomes cubic or pseudo- cubic before $T_{\mathrm{m}}$ no matter what initial starting metric symmetry they have.

\section{CONCLUSIONS}

The $\mathbf{G} \pm 1 / 2[111]^{*}$ p satellite reflections and $a^{-} a^{-} a^{-}$octahedral tilting characteristic of the $R 3 c$ rhombohedral ferroelectric phase disappear at the depolarization temperature, $T_{\mathrm{d}}$, of the rhombohedral BNTBT 4 sample. The depolarization temperature $\left(T_{\mathrm{d}}\right)$ in the BNTBT 4 sample therefore reflects a structural phase transition from a rhombohedral ferroelectric to a metrically pseudo-cubic phase, which shows relaxor characteristics in the clear frequency dependence of the dielectric constant. The poled BNTBT 12 sample is already in a metrically tetragonal phase at room temperature. The depolarization temperature $\left(T_{\mathrm{d}}\right)$ in the BNTBT 12 sample correlates well with a subtle change in metric symmetry, from metrically tetragonal to metrically cubic (or pseudo-cubic). In the case of the poled BNTBT sample in the MPB region, BNTBT 7, the dielectric properties show a quite distinct two stage transition from a room temperature clearly metrically tetragonal phase again to a metrically cubic or pseudo-cubic symmetry. Electron diffraction shows the presence of considerable octahedral tilt twin disorder in all samples.

\section{ACKNOWLEDGMENTS}

J.W., Y.L., R.L.W., Q.L., and Y.P.G. appreciate the support of the Australian Research Council (ARC) in the form of a Discovery Grant. Y.L. also appreciates support from the ARC Future Fellowships program. J.W., Y.L., and A.S. also thank the Australian Institute of Nuclear Science and Engineering (ANSIE) for financial support to access the national neutron facilities at ANSTO. 
${ }^{1}$ Y. P. Guo, Y. Liu, R. L. Withers, F. Brink, and H. Chen, Chem. Mater. 23, 219 (2011).

${ }^{2}$ W. W. Ge, J. F. Li, H. Cao, D. Viehland. Q. H. Zhang, and H. S. Luo, Appl. Phys. Lett. 95, 162903 (2009).

${ }^{3}$ W. W. Ge, H. Liu, X. G. Zhao, X. B. Li, X. M. Pan, D. Lin, H. Q. Xu, X. P. Jiang, and H. S. Luo, Appl. Phys. A 95, 761 (2009).

${ }^{4}$ W. W Ge, J. J. Yao, C. DeVreugd, J. F. Li, D. Viehland, Q. H. Zhang, and H. S. Luo, Solid State Commun. 151, 71 (2011).

${ }^{5}$ C. Ma and X. Tan, Solid State Commun. 150, 1497 (2010).

${ }^{6}$ V. Dorcet and G. Trolliard, Acta Mater. 56, 1753 (2008).

${ }^{7}$ V. Dorcet, G. Trolliard, and P. Boullay, J. Magnet. Magnet. Mater. 321, 1758 (2009).

${ }^{8}$ V. Dorcet, G. Trolliard, and P. Boullay, Chem. Mater. 20, 5061 (2008).
${ }^{9}$ G. Trolliard and V. Dorcet, Chem. Mater. 20, 5074 (2008).

${ }^{10}$ D. Lin and K.W. Kwok, Appl. Phys. A 97, 229 (2009).

${ }^{11}$ C. G. Xu, D. Lin, and K. W. Kwok, Solid State Sci. 10, 934 (2008).

${ }^{12}$ M. Chen, Q. Xu, B. H. Kim, B. K. Ahn, J. H. Ko, W. J. Kang, and O. J. Nam, J. Eur. Ceram. Soc. 28, 843 (2008).

${ }^{13}$ J. E. Daniels, W. Jo, J. Rödel, and J. L. Jones, Appl. Phys. Lett. 95, 032904 (2009).

${ }^{14}$ H. Simons, J. Daniels, J. Wook, R. Dittmer, A. Studer, M. Avdeev, J. Rodel, and M. Hoffman, Appl. Phys. Lett. 98, 082901 (2011).

${ }^{15}$ J. Kling, S. Hayn, L. A. Schmitt, M. Groting, H. J. Kleebe, and K. Albe, J. Appl. Phys. 107, 114113 (2010).

${ }^{16}$ L. A. Schmitt and H.-J. Kleebe, Func. Mater. Lett. 3, 55 (2010).

${ }^{17}$ M. Hinterstein, M. Knapp, M. Hölzel, W. Jo, A. Cervellino, H. Ehrenberg, and H. Fuessa, J. Appl. Cryst. 43, 1314 (2010). 